\title{
Cometary nature of the 1908 Tunguska cosmic body
}

\author{
F. S. Ibodov ${ }^{1}$, S. S. Grigorian ${ }^{1}$, and S. Ibadov ${ }^{2}$ \\ ${ }^{1}$ Moscow State University, Moscow, Russia, \\ emails mshtf@sai.msu.ru, firuz@pochta.ru, grigor@imec.msu.ru \\ ${ }^{2}$ Institute of Astrophysics, Dushanbe, Tajikistan \\ email: ibadovsu@yandex.ru
}

\begin{abstract}
The cometary nature of the 1908 Tunguska cosmic body is compatible with the predictions of an analytical theory of the 1908 Tunguska explosion developed in 1976-1979. The theory takes into account the three simultaneously occurring processes, namely aerodynamic destruction of the cosmic body in the Earth's atmosphere, transversal expansion of the crushed mass under the action of pressure gradient on the frontal surface of the body, and an aerodynamic deceleration of crushed expanding mass. The use, for the mechanical parameters of the Tunguska cosmic body, of the characteristics of a cometary nuclei such as that of comet Halley 1986 III and comet Shoemaker - Levy 9 1994, gives parameters of the Tunguska explosion derived from observations of Tunguska event in the Siberian taiga in 1908.
\end{abstract}

Keywords. comets: general; flares: comet nuclei, planetary atmospheres, aerodynamic destruction, transversal expansion, Tunguska explosion

\section{Introduction}

The nature of the Tunguska explosion occurred in Siberian taiga, Russia, on June 30, 1908, that produced catastrophic destruction in the taiga, remained mysterious during many decades, and scientific investigations were carried out to accumulate data on the parameters of this event. The estimation of the initial mass of the exploded cosmic body $M_{o}$ made on the basis of observations of reduction of solar radiation due to atmospheric opacity in California, USA, is of the order of $10^{6}$ tons (Fesenkov 1949). From the analysis of crushing the taiga, the derived energy of explosion was of the order of $E_{e}=4 x 10^{24} \mathrm{erg}$ or 10 megatons in TNT equivalent, i.e., 1000 times the energy of the Hiroshima atomic explosion. The geocentric velocity of the bolide being considered as a fragment of comet Encke, is about $30 \mathrm{~km} / \mathrm{s}$, the inclination angle of his flight trajectory to the horizon is $\alpha=30-45^{\circ}$, the altitude of explosion of the bolide above the Earth's surface is estimated as $h_{e}=5-10 \mathrm{~km}$ (Korobeinikov et al. 1991, and references therein).

\section{Analytical theories of explosion of Tunguska cosmic body}

The hypothesis of the Tunguska body as a cosmic body of anomaly small density (less than $0.01 \mathrm{~g} / \mathrm{cm}^{3}$ ), for which the generation of a strong shock wave above the Earth's surface becomes possible due to the sharp aerodynamic deceleration of the evaporating body and the fully dissipation of its large initial kinetic energy in the atmosphere, was theoretically considered in 1975 . However, the theory was not applicable because of unacceptability of the starting hypothesis on the extremely low density of cosmic body (Petrov \& Stulov 1975; Grigorian 1979; Surdin et al. 1982). 
An analytical theory of the Tunguska explosion, taking into account an aerodynamic destruction of cosmic body, transverse expansion of the crushed mass under the action of pressure gradient on the frontal surface of the body and aerodynamic deceleration of the fragmented flattened expanding hypervelocity mass, was developed by Grigorian (1976, 1979).

The theory was used, applying numerical methods of integration and mathematical modeling, also for a quantitative estimation of events, occurring at the entry of fragments of the nucleus of comet Shoemaker-Levy 9 into the atmosphere of Jupiter on July 16-22, 1994 (Grigorian 1994; Fortov et al. 1996).

In 2008 we modified the theory of Grigorian (1979) to find the law of variation of the kinetic energy of the fragmented mass in the explosion zone in an explicit form, that is necessary for deriving the position of the point of maximal deceleration - maximal energy release, and solving thus the problem of analytically finding the altitude and other parameters of "explosion" of cosmic bodies like cometary nuclei, having usually small density and material strength, in the atmospheres of planets (Ibadov et al. 2008a).

The solution of the above problem is of interest also to investigate explosion of sungrazing comets in the atmosphere of the Sun and comet-like bodies, as well as stargrazers, in the atmospheres of young stars in order to study flare mechanisms of such stellar objects (Ibadov 2007; 2008b).

\section{Nature of the Tunguska cosmic body}

The basic parameter of the Tunguska event, the altitude of "explosion" of bolide in the Earth's atmosphere where the maximal energy release due to sharp aerodynamic deceleration of the crushed and transversally expanding mass occurs, is determined as

$$
z_{m}=z_{e}=z_{*}-H \ln \left(1+\frac{\sqrt{3 C_{x}} C}{2 b}\right)=H \ln \left(\frac{\rho_{o} V_{o}^{2}}{\left(1+\frac{\sqrt{3 C_{x}} C}{2 b}\right) \sigma_{*}}\right)=H \ln \left(\frac{2 b \rho_{o} V_{o}^{2}}{\sqrt{3 C_{x}} C \sigma_{*}}\right),
$$

where

$$
\begin{aligned}
& z_{*}=H \ln \left[\left(\frac{\rho_{o} V_{o}^{2}}{\sigma_{*}}\right)\right], \quad b=\nu \exp \left(-\frac{z_{*}}{H}\right), \\
& \nu=\frac{3 C_{x} \rho_{o} H}{4 \rho_{b} R_{o} \sin \alpha}, \quad C=\left(\frac{3 C_{x} R_{o} \sin \alpha}{8 H}\right)^{1 / 2}
\end{aligned}
$$

$z_{*}$ is the altitude corresponding to the onset of the cosmic body aerodynamic destruction in the Earth's atmosphere, $H$ is the height scale of the atmosphere, $R_{o}$ is the body initial radius, $V_{o}$ is the initial entry velocity of the body to the atmosphere, $\rho_{b}$ and $\sigma_{*}$ are the density and mechanical strength of the body material.

Assuming for the Tunguska cosmic body $M_{o}=4 x 10^{12} \mathrm{~g}$ (Fesenkov 1949), $\rho_{b}=1 \mathrm{~g} / \mathrm{cm}^{3}$ (Marov 1994) and $R_{o}=10^{4} \mathrm{~cm}, V_{o}=3 x 10^{6} \mathrm{~cm} / \mathrm{s}, \alpha=30^{0}, \sigma_{*}=10^{7} \mathrm{dynes} / \mathrm{cm}^{2}, C_{x}=1$, $\rho_{o}=1.3 \times 10^{-3} \mathrm{~g} / \mathrm{cm}^{3}, H=7 \times 10^{5} \mathrm{~cm}$ according to (1), (3.2) and (3.3) we obtain $\nu=0.14$, $b=1.2 x 10^{-4}, C=5.1 \times 10^{-2}, z_{*}=48.3 \times 10^{5} \mathrm{~cm}=48.3 \mathrm{~km}, z_{m}=7.3 \times 10^{5} \mathrm{~cm}=7.3 \mathrm{~km}$.

It should be noted that the theoretical values of "explosion" altitude of the cosmic body $z_{m}$, computed with (1) will correspond to the altitude of explosion $h_{e}$ obtained from observations of the Tunguska phenomenon as well as to the mass and the radius of the cosmic body $M_{o}$ and $R_{o}$ of the order of $10^{12} \mathrm{~g}$ and $100 \mathrm{~m}$, respectively, to the initial kinetic energy of the body $E_{o}$ in the range of $10^{24}-10^{25}$ erg in the case if the density of the body is $\rho_{b}=0.5-1 \mathrm{~g} / \mathrm{cm}^{3}$. 
The obtained density of the exploded Tunguska cosmic body is characteristic for the density of nuclei of comets (Reinhard 1986; Sagdeev 1986; Fortov et al. 1996).

\section{Conclusions}

The theory of aerodynamic destruction of cosmic bodies and transversal expansion of fragmented mass in the Earth's atmosphere can adequately explain the 1908 Tunguska explosion for mechanical parameters of the body like those for comet Halley 1986 III and comet Shoemaker - Levy 1994. It indicates, along with data of observations of the phenomena, that the 1908 Tunguska cosmic body had cometary nature.

\section{Acknowledgements}

The authors are grateful to Academician A. M. Cherepashchuk for his interest and stimulating remarks and also to Drs. V.F. Esipov and G.M. Rudnitskij, Sternberg Astronomical Institute of Moscow State University for their promotion in fulfilling the research. The work is supported by the Russian Foundation for Basic Research, Project RFBR 08-02-11003-ano.

\section{References}

Fesenkov, V. G. 1949, Meteoritika, 6, 8

Grigorian, S. S. 1976, Dokl. Akad. Nauk SSSR, 231, 57

Grigorian, S. S. 1979, Kosmich. Issled., 17, 875

Grigorian, S. S. 1994, Dokl. Akad. Nauk, 338, 752

Ibadov, S., Ibodov, F. S., \& Grigorian, S. S. 2008a, in: Intern. Conf. "100 Years Since Tunguska Phenomenon: Past, Present and Future" // tunguska.sai.msu.ru/index.php?q=present

Ibadov, S., Ibodov, F. S. \& Grigorian, S. S. 2007, in: Star-Disk Interaction in Young Stars, Proc. IAU Symp. No. 243, Grenoble, France, p. VI.4 // www.iaus243.org

Ibadov, S., Ibodov, F. S. \& Grigorian, S. S. 2008b, in: Universal Heliophysical Processes, Proc. IAU Symp. No. 257, Cambridge University Press, 341

Korobeinikov, V. P., Chushkin, P. I., \& Shurshalov, L. V. 1991, Astron. Vestnik, 25, 327

Marov, M. Ya. 1994, Astron. Vestnik, 28, 5

Petrov, G. I. \& Stulov, V. P. 1975, Kosmich. Issled. (Letters), 13, 587

Reinhard, R. 1986, Nature, 321, 313

Sagdeev, R. Z., Blamont, J., Galeev, A. A., Moroz, V. I., Shapiro, V. D., Shevchenko, V. I., \& Szego, K. 1986, Nature, 321, 259

Surdin, V. G, Romeiko, V. A, \& Koval, V. I. 1982, Astron. Circular USSR, 1206, 1 